\title{
Top to Bottom: A New Method for Assessing Adequacy of Laparoscopic Pyloromyotomy
}

\author{
Maria E. Linnaus, MD, ${ }^{1,2}$ Crystal S. Langlais, MPH, Kevin N. Johnson, MD, and David M. Notrica, MD ${ }^{1,2}$
}

\section{Abstract}

Introduction: Hypertrophic pyloric stenosis is a commonly encountered pediatric surgical issue. Initially treated with open surgical techniques, many pediatric surgeons have adopted the minimally invasive approach using laparoscopy. However, some concerns exist that the rate of incomplete pyloromyotomy is elevated in laparoscopy. We propose a new technique to assess the adequacy of laparoscopic pyloromyotomy.

Methods: Adequacy of laparoscopic pyloromyotomy was assessed by confirming that the top of the serosa on one side of the pylorus has adequate freedom to reach the bottom of the muscle on other side. A retrospective review of patients undergoing laparoscopic pyloromyotomy confirmed by this method from March 2012 to January 2016 was conducted. Demographics, laboratory values on admission, and postoperative outcomes were collected. Descriptive statistics was utilized.

Results: Thirty-three patients were included. Median age was 30 days (interquartile range [IQR]: 24, 47). Median pylorus length and thickness were $19 \mathrm{~mm}$ (IQR 17.3, 21) and $4.5 \mathrm{~mm}$ (IQR: 4.0, 4.8), respectively. Median time to first full feed was 8.5 hours (IQR: 6.6, 15.6). Twenty-three $(69 \%)$ patients had postoperative emesis. Median length of stay postoperation was 26.5 hours (IQR: 21.1, 44.7). There were no reoperations for incomplete pyloromyotomy and no infections. On follow-up, 1 patient had prolonged postoperative emesis that resolved without further intervention and 1 patient on peritoneal dialysis before surgery had an incisional hernia that required operation in the setting of bilateral inguinal hernias.

Conclusion: In a small series, the top to bottom assessment appears to confirm adequacy of pyloromyotomy.

Keywords: hypertrophic pyloric stenosis, incomplete pyloromyotomy, laparoscopic pyloromyotomy, technique

\section{Introduction}

$\mathbf{H}$ YPERTROPHIC PYLORIC STENOSIS (HPS) is one of the mOSt common conditions affecting newborns (Fig. 1). ${ }^{1}$ It has long been recognized as a surgical condition since the late 1800s, but was treated with extensive surgery until the advent of the pyloromyotomy in $1911 .^{2}$ Later, a transumbilical approach, and subsequently, the laparoscopic pyloromyotomy in 1991 were described. ${ }^{3}$ Before laparoscopy, surgeons utilized manual assessment of independent mobilization of the two edges of the myotomy to slide as a mode for determining adequacy of pyloromyotomy. ${ }^{2,4}$ However, with the increasing use of laparoscopic techniques, concerns have arisen that there may be an increased risk of incomplete pyloromyotomy with the laparoscopic approach. ${ }^{5-7}$ Several randomized trials and meta-analyses have demonstrated superiority of the laparoscopic approach in decreasing length of hospitalization and time to full feeds and improving cosmesis without significant differences in rates of mucosal perforation, incisional hernia, or wound infections. ${ }^{6,8-10}$

As part of the effort to combat the issue of incomplete pyloromyotomy, surgeons have attempted to utilize new techniques to minimize this risk. In 2004, Ostlie et al. proposed standardizing the length of pyloromyotomy to $2 \mathrm{~cm}$ as a means of avoiding incomplete pyloromyotomy during laparoscopic surgery. ${ }^{11}$ Using that technique, the authors had a $0 \%$ incidence of incomplete pyloromyotomy without any mucosal perforations or duodenal injuries. ${ }^{11}$ However, this technique requires measurement of the length and does not account for anatomic variability. Therefore, this has the potential to lead to inadequate pyloromyotomy in cases of an abnormally long pyloric channel. We propose a simplified technique to determine the adequacy of pyloromyotomy during laparoscopy.

\footnotetext{
${ }^{1}$ Division of Pediatric Surgery, Phoenix Children's Hospital, Phoenix, Arizona.

${ }^{2}$ Department of Surgery, Mayo Clinic Hospital, Phoenix, Arizona.

${ }^{3}$ Department of Pediatric Surgery, Mott Children's Hospital, University of Michigan, Ann Arbor, Michigan.
} 


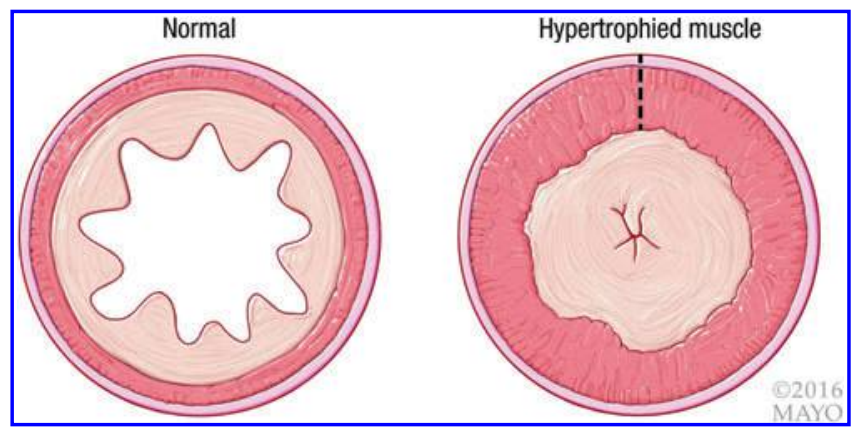

FIG. 1. Cross-sectional view of normal pyloric mucosa (left) and hypertrophic pyloric stenosis (right). By permission of Mayo Foundation for Medical Education and Research. All rights reserved.

\section{Patients and Methods}

A retrospective review was performed of all laparoscopic pyloromyotomy cases performed by a single surgeon (DMN) from March 2012 through January 2016 using the proposed method (detailed below). Variables collected included demographics, family history of pyloromyotomy, laboratory values on admission to the hospital, operative time, postoperative complications, such as episodes of emesis, and recurrence rate due to incomplete pyloromyotomy. Descriptive statistics was reported using STATA 13.1 (College Station, TX). Continuous variables are reported as median and interquartile range (IQR) and range, where appropriate.

\section{Operative Technique}

After induction of general anesthesia, a $3 \mathrm{~mm}$ vertical incision in the inferior aspect of the umbilicus is made, and utilizing a hemostat, the typically unclosed natural umbilical opening is located and entered; the fascia is stretched to allow for trocar entry. A $3 \mathrm{~mm}$ trocar is inserted into the umbilicus for camera placement. While the assistant holds the camera and after infiltration of local anesthesia, two stab incisions utilizing a 15blade scalpel are placed in line with the umbilicus laterally to the right and left as the working ports under laparoscopic guidance. Trocarless entry is then used with $3 \mathrm{~mm}$ instruments. The pylorus is grasped through the left-sided incision using a pyloric spreader and brought anteriorly with a slight rotation to orient the pylorus vertically to improve the view.

Next, utilizing a protected-tip medium Teflon-coated electrocautery blade extender (4-inch, extended blade electrode modified, catalog \#0014AM; Megadyne Medical Products, Draper, UT), the pyloric serosa is scored to a $1 \mathrm{~mm}$ depth without entry into the muscle below. Still utilizing the "cold" Bovie (without electrocautery), the muscle is then bluntly disrupted along the length of the pylorus by rubbing along the scored serosa until the outer mucosa is reached. The pyloric muscle is grasped, and counter traction with the broad edge of the bovie is used to provide a slow steady separation of the hypertrophic in the middle of the myotomy with the mucosa under direct vision.

To assess the adequacy of the pyloromyotomy, the two cut edges of the pylorus are checked. The cephalad portion of incised pylorus is elevated and the inferior portion is depressed toward the mucosa (Fig. 2). If the myotomy is adequate, the top of the serosa on the inferior portion of the incised pylorus will reach the bottom of the submucosa on the superior portion of the incised pylorus. Figure 3 demonstrates an inadequate pyloromyotomy where the top of the serosa does not fully reach the bottom of the muscle. In this situation, repeated separation continues until the "top to bottom" assessment is achieved.

The instruments are removed from the lateral abdomen and the $3 \mathrm{~mm}$ umbilical site trocar is removed. The umbilical fascia is closed with an absorbable braided suture and all skin is closed with skin glue.

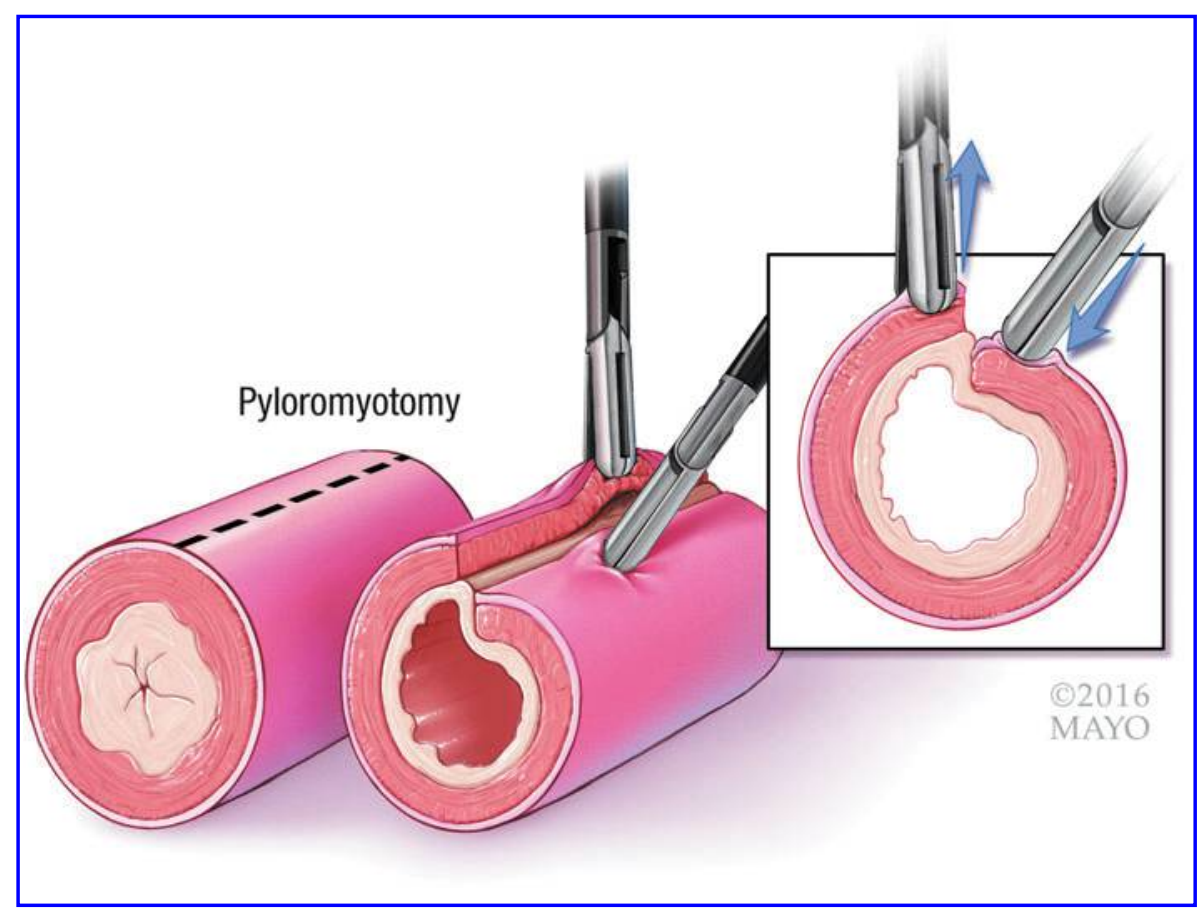

FIG. 2. Demonstration of the "top to bottom" technique during laparoscopic pyloromyotomy. By permission of Mayo Foundation for Medical Education and Research. All rights reserved. 


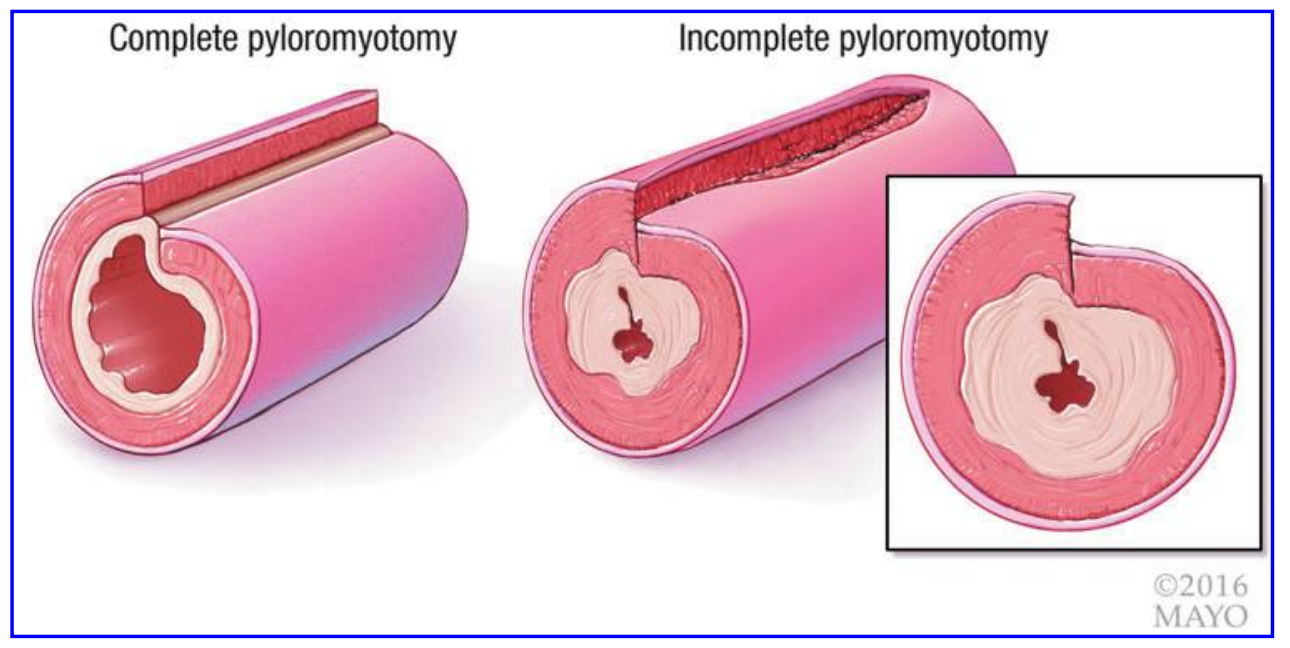

FIG. 3. Complete pyloromyotomy (left) compared to an example of incomplete pyloromyotomy (right). By permission of Mayo Foundation for Medical Education and Research. All rights reserved.

Postoperatively, patients are allowed to eat ad libitum feeds, often breast milk or formula. At our institution, patients are discharged upon tolerating two $60 \mathrm{~mL}$ feeds with less than $5 \mathrm{~mL}$ of emesis.

\section{Results}

During the study period, 33 patients met inclusion; 28 (85\%) were male and most were either Hispanic $(21 ; 64 \%)$ or white $(9 ; 27 \%)$. The median age at time of surgery was 30 days (IQR: 24, 47; range: $18-107)$. The majority of patients were not firstborn $(n=18 ; 55 \%)$; three of these patients had a sibling with a known history of HPS ( 2 brothers, 1 sister).

Median (IQR) laboratory evaluations on admission were as follows: sodium: 139 (137, 140), potassium: $4.8(4.5,5.1)$, chloride: 103.5 (99.5, 105.5), carbon dioxide: $27(25.5,28)$, and glucose: $80(75,86)$. The median length and thickness of the pylorus, as measured preoperatively by ultrasound, were $19 \mathrm{~mm}$ (IQR: 17.3, 21; range: $14-24$ ) and $4.5 \mathrm{~mm}$ (IQR: 4, 4.8; range: 3.4-7.7), respectively. Median (IQR) operative time was 22 minutes (IQR: 19, 27).

Postoperatively, median time to first feed and first full feed was 2 hours (IQR: 1.2, 3.1) and 8.5 hours (IQR: 6.6, 15.6), respectively. Twenty-three $(69.7 \%)$ patients had at least one episode of postoperative emesis; median episodes of emesis for these patients were 2 (IQR: 1,5). Median total hospital length of stay was 37.2 hours (IQR: 24.2, 64.8), while median postoperative length of stay was 26.5 hours (IQR: 21.1, 44.7).

\section{Complications and follow-up}

There were no postoperative complications observed in this study, including no infections and no reoperations for incomplete pyloromyotomy. Twenty-two (67\%) patients returned for a follow-up visit, a median of 16 days (IQR: 14, 21; range: $10-29$ ) post discharge. Two of these patients had a complication noted. One patient had emesis at 1 month followup, not requiring hospitalization, and which resolved at second follow-up 30 days later with no intervention. The second patient was on peritoneal dialysis for congenital dysplastic kidneys and had an asymptomatic umbilical incisional hernia, which was later repaired concurrently along with asymptomatic bilateral inguinal hernias.

\section{Discussion}

The "top to bottom" approach for determining adequacy during laparoscopic pyloromyotomy has been successful in preventing inadequate pyloromyotomy in this cohort. A $0 \%$ incidence of incomplete pyloromyotomy has been demonstrated in other reports; ${ }^{11-13}$ however, this has been generally nonreproducible in most large retrospective cohort studies and randomized trials between the laparoscopic and open techniques. 5,6,14 Currently, the two general techniques to determine adequate myotomy include standardizing the length to $2 \mathrm{~cm}$ or ensuring that the two edges of the myotomy move independent of each other. ${ }^{11,15}$ In this cohort, $25 \%$ of the pyloric channel lengths were $>2.1 \mathrm{~cm}$ and the longest pyloric length was $2.4 \mathrm{~cm}$; thus, standardizing the length to $2 \mathrm{~cm}$ may not be adequate for all patients. In addition, for studies utilizing the independent movement of the two sides as an indicator of adequacy, most rates of incomplete pyloromyotomy are not zero. The authors believe this is due to difficulty assessing transverse sliding when using a laparoscopic approach. In a recent, large multicenter retrospective review, the authors demonstrated a $1.2 \%$ incidence of incomplete pyloromyotomy in the laparoscopic approach compared to a $0.3 \%$ incidence in the open approach. ${ }^{14}$ In prospective randomized controlled trials and meta-analyses between the open and laparoscopic approach, a 3\%-6\% risk of incomplete myotomy was found in the laparoscopic groups. ${ }^{5-7}$ This questions whether independent sliding of the superior and inferior portions of the pyloromyotomy alone can be adequately assessed in the laparoscopic setting without the tactile feedback obtained through an open incision.

The "top to bottom" technique is independent of some of the anatomic variability between patients and is a more reproducible approach compared to other previously described techniques. ${ }^{15}$ In addition, this technique adds no time to the operation. The operative time of 22 minutes in this cohort was comparable to most other laparoscopic studies reporting 20-30 minutes or less for operative time. $5,12,16,17$

Postoperative emesis is expected after pyloromyotomy. In this cohort, $\sim 70 \%$ of patients had at least one episode of emesis in the postoperative period, yet no children required return to the operating room for repeat pyloromyotomy or 
required readmission for persistent emesis. In other studies looking at the incidence of postoperative emesis, the majority of infants had at least one episode or more. ${ }^{16,18,19}$ Only one patient in this group had persistent emesis at follow-up and underwent additional imaging for concerns of incomplete pyloromyotomy. However, upper GI examination was negative for recurrent pyloric stenosis, incomplete myotomy, or another pathology. This child's emesis resolved with reflux medications without intervention. There were no wound infections, mucosal injuries, or duodenal injuries. The one patient who developed an incisional hernia had congenital dysplastic kidneys and was on peritoneal dialysis before developing pyloric stenosis.

Despite episodes of emesis, patients may safely continue feeding. In this cohort, the average patient reached their full feeding regimen by 8.5 hours. The 2 -hour time to initial postoperative feeds was also comparable to the feeding regimens that have been proposed by other centers. ${ }^{18}$ Patients in our cohort were discharged from the hospital following toleration of two full feeds, which was around 26.5 hours after operation in the average patient. This time to discharge was similar to other reports. ${ }^{16,18,19}$

\section{Limitations}

As in any retrospective study, there are weaknesses that limit the conclusions. Primarily, this cohort size is small and only one surgeon utilized this technique during the study period. Additional studies with an increased number of patients undergoing pyloromyotomy utilizing this technique may be necessary to determine whether the incidence of incomplete pyloromyotomy is lower than in previously described techniques. In addition, retrospective chart review limited the authors' ability to determine the exact volume of emesis, and therefore, number of episodes was reported; volume may have been a better indicator of how well the patient is tolerating feeds.

\section{Conclusion}

The "top to bottom" approach for determining adequacy of laparoscopic pyloromyotomy is a visual technique. The technique is associated with good outcomes, similar to other reports of laparoscopic pyloromyotomy. Potential advantages of this technique are its objectivity, visual demonstrability, utility independent of pyloric channel length, and ease of adoption.

\section{Acknowledgment}

The authors acknowledge Frank Corl for his assistance with the illustrations exhibited in this article.

\section{Disclosure Statement}

No competing financial interests exist.

\section{References}

1. Schechter R, Torfs CP, Bateson TF. The epidemiology of infantile hypertrophic pyloric stenosis. Paediatr Perinat Epidemiol 1997;11:407-427.

2. Taylor S. Pyloric stenosis before and after Ramstedt. $\underline{\text { Arch }}$ Dis Child 1959;34:20-23.

3. Alain JL, Grousseau D, Terrier G. Extramucosal pyloromyotomy by laparoscopy. Surg Endosc 1991;5:174-175.

4. Koontz CS, Wulkan ML. Lesions of the stomach. In: Holcomb GW, 3rd, Murphy JP, Ostlie DJ (eds). Ashcraft's
Pediatric Surgery, Sixth ed. Saunders Elsevier, Inc., Amsterdam, The Netherlands, 2014, pp. 403-413.

5. Leclair MD, Plattner V, Mirallie E, et al. Laparoscopic pyloromyotomy for hypertrophic pyloric stenosis: A prospective, randomized controlled trial. J Pediatr Surg 2007; 42:692-698.

6. Hall NJ, Pacilli M, Eaton S, et al. Recovery after open versus laparoscopic pyloromyotomy for pyloric stenosis: A double-blind multicentre randomised controlled trial. Lancet 2009;373:390-398.

7. Jia W-Q, Tian J-H, Yang K-H, et al. Open versus laparoscopic pyloromyotomy for pyloric stenosis: A metaanalysis of randomized controlled trials. Eur J Pediatr Surg 2011;21:77-81.

8. Oomen MW, Hoekstra LT, Bakx R, Ubbink DT, Heij HA. Open versus laparoscopic pyloromyotomy for hypertrophic pyloric stenosis: A systematic review and meta-analysis focusing on major complications. Surg Endosc 2012;26: 2104-2110.

9. Siddiqui S, Heidel RE, Angel CA, Kennedy AP Jr. Pyloromyotomy: Randomized control trial of laparoscopic vs. open technique. J Pediatr Surg 2012;47:93-98.

10. Sola JE, Neville HL. Laparoscopic vs. open pyloromyotomy: Asystematic review and meta-analysis. $\underline{\mathrm{J} \text { Pe- }}$ diatr Surg 2009;44:1631-1637.

11. Ostlie DJ, Woodall CE, Wade KR, et al. An effective pyloromyotomy length in infants undergoing laparoscopic pyloromyotomy. Surgery 2004; 136:827-832.

12. St Peter SD, Holcomb GW, 3rd, Calkins CM, et al. Open versus laparoscopic pyloromyotomy for pyloric stenosis: A prospective, randomized trial. Ann Surg 2006;244:363-370.

13. Anwar MO, Al Omran Y, Al-Hindi S. Laparoscopic pyloromyotomy: A modified simple technique. J Neonatal Surg 2016;5:3-6.

14. Hall NJ, Eaton S, Seims A, et al. Risk of incomplete pyloromyotomy and mucosal perforation in open and laparoscopic pyloromyotomy. J Pediatr Surg 2014;49:1083-1086.

15. Thomas PG, Sharp NE, St Peter SD. Laparoscopic pyloromyotomy: Comparing the arthrotomy knife to the Bovie blade. J Surg Res 2014;190:251-254.

16. St Peter SD, Tsao K, Sharp SW, Holcomb GW, 3rd, Ostlie DJ. Predictors of emesis and time to goal intake after pyloromyotomy: Analysis from a prospective trial. J Pediatr Surg 2008;43:2038-2041.

17. Yagmurlu A, Barnhart DC, Vernon A, Georgeson KE, Harmon CM. Comparison of the incidence of complications in open and laparoscopic pyloromyotomy: A concurrent single institution series. J Pediatr Surg 2004;39:292-296.

18. Adibe OO, Iqbal CW, Sharp SW, et al. Protocol versus ad libitum feeds after laparoscopic pyloromyotomy: A prospective randomized trial. J Pediatr Surg 2014;49:129-132; discussion 132.

19. Perger L, Fuchs JR, Komidar L, Mooney DP. Impact of surgical approach on outcome in 622 consecutive pyloromyotomies at a pediatric teaching institution. J Pediatr Surg 2009;44:2119-2125.

Address correspondence to: David M. Notrica, MD Division of Pediatric Surgery Phoenix Children's Hospital 1919 E Thomas $R d$ Phoenix, AZ 85016

E-mail: dnotrica@phoenixchildrens.com 
This article has been cited by:

1. Sherwin S. Chiu, James C. Gilbert. 2018. Recurrent pyloric stenosis: a form of the incomplete pyloromyotomy. Journal of Pediatric Surgery Case Reports 29, 14-17. [Crossref] 